\title{
Digital Internal Module Controller Shaped by Sensitivity Function of Platform Driven by Ultrasonic Motor
}

\author{
Pan Song ${ }^{1 *}$, Xu Zhang-Fan ${ }^{1}$, Huang Wei-Qing ${ }^{2}$ \\ ${ }^{1}$ State Key Laboratory of Mechanics and Control of Mechanical Structures, Nanjing University of \\ Aeronautics and Astronautics, 210016 Nanjing, China. \\ ${ }^{2}$ School of Mechanical and Electrics Engineering, Guangzhou University, 510006 Guangzhou, China. \\ * Corresponding author. Tel.: +86 3914711165; email: pansong@nuaa.edu.cn \\ Manuscript submitted October 25, 2017; accepted November 10, 2017. \\ doi: 10.17706/ijapm.2018.8.3.45-52
}

\begin{abstract}
When the ultrasonic motor is used to drive a stabilized platform, there will be variable load are applied on the motor. So the motor's motion state is changed. It is necessary to carry out some relative researches on the problem. Firstly, this paper establishes the dynamics model of stabilized platform driven by the ultrasonic motor. Secondly, combined with the established model, the internal model controller is designed to satisfy the stability requirement of the stabilized platform from the disturbance to the output. In order to verify the control effect of the proposed controller, the control effect of the proposed controller is simulated and compared with the Proportion-Integral-Derivative controller. We can find that this control method is better than classical Proportion-Integral-Derivative controller. The unit step response time reduces $0.03 \mathrm{~s}$. The tracking error of $1 \mathrm{~Hz}$ sinusoidal reference signal reduces from $1.9 \%$ to $0.6 \%$. The ability of restraining the external disturbance improves about $10 \mathrm{~dB}$ and the suppression band at about $-30 \mathrm{~dB}$ also increased by about $4 \mathrm{~Hz}$.
\end{abstract}

Key words: Ultrasonic motor, stabilized platform, sensitivity function, digital control.

\section{Introduction}

The stabilized platform is an auto-control system [1] which can be used to eliminate disturbance and to track a target through velocity control. This equipment has found a wide use in many applications such as the image processing, guide missile and tracking system. Nowadays much attention has been paid on the tracking accurateness and the response. Meanwhile the miniaturization and integration are also required [2]. Recently, ultrasonic motor (USM) have attracted considerable attention as a new type of actuator in servo-systems [3]. The USM can provide high driving torque at low rotational speed and high holding torque. The mass of the USM is smaller compared with the electromagnetic motor.

The principle of the USM is based on the converse piezoelectric effect for converting electrical power into mechanical vibrations, which cause the rotor to move [4]. The speed and torque of the USM is related with the voltage, frequency and the phase difference between the two sinusoidal voltages. In this paper, a method with fixed voltage, fixed phase difference and variable frequency is adopted.

At present, most of the stabilized platforms use electromagnetic motor as their actuator, and the control methods are suit for this kind of platform. A fuzzy-classic controller to reduce the overshoot is presented in [5]. A sliding mode variable structure controller is described in [6]. A fuzzy self-adaptation Proportion-Integral-Derivative (PID) controller which can reduce the overshoot as well as improve the 
tracking accuracy can be found in [7]. In [8], an improved robust inner module controller is used for disturbance rejection over a wide frequency band. Recently, considering the limitations of classical control methods, many modern control theories and intelligent control methods are under consideration. Beside the fuzzy controller [9] and neural network [10], some compound controller such as fuzzy-neural network control is presented [11]. The control methods in [9]-[11] are proposed to control the rotation speed without load. This is different from the aim of stabilized platform which is mostly used to suppress disturbance, and the load on the motor is variable.

A main parameter to evaluate the performance of stabilized platform is isolation. When the base is under sinusoidal movement, it can be calculated by dividing the amplitude of device space oscillation and the amplitude of sinusoidal movement [12]. In this paper, a controller is designed to satisfy the requirement of isolation by shape the sensitivity function of the control system.

\section{Platform Driven by Ultrasonic Motor}

A schematic diagram of three axis stable platform is shown in Fig. 1.

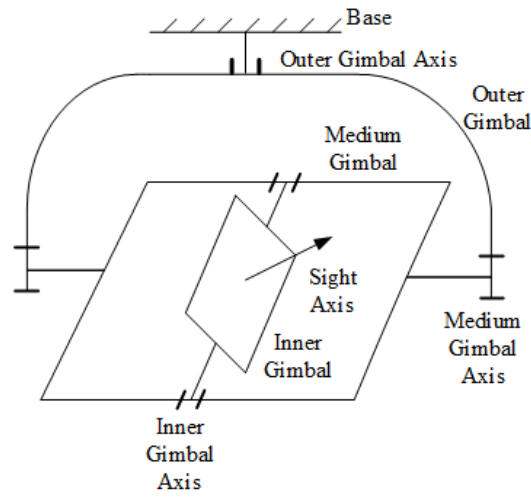

Fig. 1. Schematic diagram of three axis stable platform.

The three axis stable platform consists of inner, medium, outer gimbals and the actuators are installed on the gimbals. The sighting axis on the inner gimbal is able to maintain stability by the three gimbals cooperative rotate.

Take the inner gimbal as an example, the motion equation of this gimbal is

$$
M_{e l}-M_{d p}-M_{i m b p x}=J_{p x} \dot{\omega}_{p x}
$$

where $M_{e l}$ is the driving torque of the USM, $M_{d p}$ is the unknown disturbance torque, $M_{i m b p x}$ is the projection component of disturbance torque caused by the unbalanced mass, $J_{p x}$ is the inner gimbal inertia, $\dot{\omega}_{p x}$ is the angular velocity of inner gimbal.

The unknown disturbance torque in (1) is

$$
M_{d p}=M_{f p}+M_{c r p}+k_{f p} \dot{\varepsilon}+k_{c r p} \dot{\varepsilon}
$$

where $M_{f p}$ is the friction torque, $M_{c r p}$ is the cable flexible disturbance torque, $k_{f p}$ is the friction coefficient, $k_{c r p}$ is the cable flexible disturbance coefficient.

The disturbance torque caused by the unbalanced mass of inner gimbal is

$$
M_{i m b p x}=\left(J_{p z}-J_{p y}\right) \dot{\omega}_{p y} \dot{\omega}_{p z}
$$


This torque is caused by gyroscopic effect. When the inner gimbal inertia of three axis are equal, $J_{p x}=J_{p y}=J_{p z}$ The kinetic equation will be simplified as (4).

$$
M_{e l}-M_{d p}=J_{p x} \dot{\omega}_{p x}
$$

The driving torque of the USM can be described by linear approximation [13], [14].

$$
M_{e l}=f_{0}\left(w_{i d}-w\right)
$$

where $w$ is the actual speed. In this mathematical model, it's equal to the speed of inner gimbal, $w_{i d}$ is the ideal speed, $f_{0}$ is the coefficient accessed by system identification.

There is a dead zone exists in the speed characteristic of the USM. So the ideal speed of the USM is

$$
\begin{cases}w_{i d}=(2 \pi f) k \frac{h}{b^{2}} \sin (\varphi)\left(W-W_{T H}\right) & W>W_{T H} \\ w_{i d}=0 & 0<W<W_{T H}\end{cases}
$$

where $f$ is the frequency, $W$ is the amplitude of the stator's vibration, $\varphi$ is the phase difference of the voltage, $k$ is the number of the nodal diameter, $b$ is the diameter of the stator, $h$ is the thickness of the stator, $W_{T H}$ is the dead zone amplitude.

The simulation parameters are shown in Table 1.

Table 1. Simulation Parameters

\begin{tabular}{ll}
\hline Parameters & Values \\
\hline$M_{f p} / N \cdot m$ & 0.005 \\
$M_{c r p} / N \cdot m$ & 0.005 \\
$k_{f p} / N \cdot m \cdot s / r a d$ & 0.008 \\
$k_{c r p} / N \cdot m / r a d$ & 0.008 \\
$J_{p x} / N \cdot m \cdot s^{2} / \mathrm{rad}$ & 0.00171 \\
$W_{T H} / \mu m$ & 0.70276 \\
$W / \mu m$ & 0.70278 \\
$k h / b^{\wedge} 2 / m^{-1}$ & 0.05 \\
$\varphi / r a d$ & $\pi / 2$ \\
\hline
\end{tabular}

\section{Design of Digital Controller}

A standard digital controller of 'RST' is used to adjust the frequency, which is shown in Fig. 2. The polynomial $T, S$ and $R$ satisfy the format $T\left(z^{-1}\right)=t_{0}+t_{1} Z^{-1}+t_{2} Z^{2}+\ldots, \quad S\left(z^{-1}\right)=s_{0}+s_{1} Z^{-1}+s_{2} Z^{-2}+\ldots$, $R\left(z^{-1}\right)=r_{0}+r_{1} Z^{-1}+r_{2} Z^{-2}+\ldots$

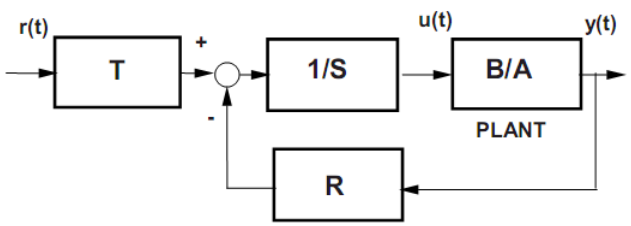

Fig. 2. Structure diagram of standard RST controller.

The block diagram of speed control system for the platform is shown in Fig. 3. The control object is to keep the sight stable through frequency control of the USM. 


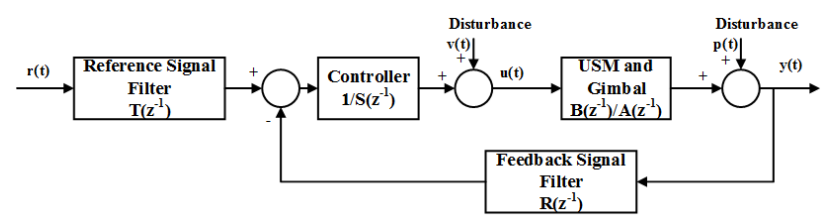

Fig. 3. Block diagram of speed control system.

Substituting values shown in table 1 into equation (4), the mathematical relation of signal's frequency and inner gimbal rotation speed would be obtained. The sample frequency is $1 \mathrm{kHz}$, and the transfer function is

$$
\frac{B\left(z^{-1}\right)}{A\left(z^{-1}\right)}=\frac{0.06278 z^{-1}-0.06278 z^{-2}}{1-1.059 z^{-1}+0.05913 z^{-2}}
$$

In this paper, this transfer function would be taken as an example to design the RST controller.

For the stabilized platform driven by USM, the function requirements are as follows:

(1) The frequency of the disturbance signal is less than $5 \mathrm{~Hz}$, the isolation should be no more than - $30 \mathrm{~dB}$;

(2) The disturbance signal is high frequency, the requirement for the isolation can be related.

The module margin is more than $6 \mathrm{~dB}$ and the delay margin are more than one sampling period [15].

The isolation of platform is the amplitude of device space oscillation over the amplitude of sine movement of disturbance. This value equals to the amplitude of the close sensitivity function which links the disturbance to the output. The performance of platform was transformed to the amplitude of sensitivity. And the performance requirements have been also transformed to the desired template for the module of sensitivity function.

The desired template for the module of sensitivity function is shown as follow:

(1) The amplitude of sensitivity function with frequency less than $5 \mathrm{~Hz}$ should be no more than $-30 \mathrm{~dB}$.

(2) The amplitude of sensitivity function in all frequency should be no more than $6 \mathrm{~dB}$.

(3) The amplitude of sensitivity function should within the scope of $1-\left|1-z^{-1}\right|^{-1}$ and $1+\left|1-z^{-1}\right|^{-1}$.

In order to improve the robustness at high frequency, the poles of controlled object are taken as the desired leading poles. The inner module of object is introduced. The improved RST inner module controller is as Fig. 4.

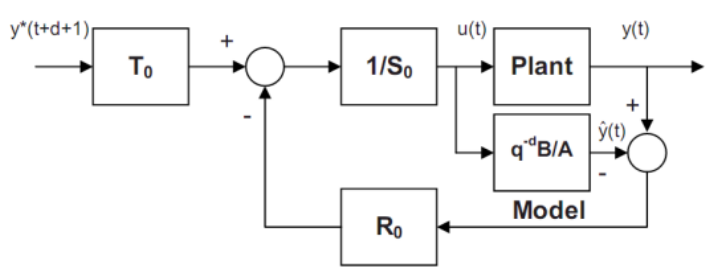

Fig. 4. Improved RST internal module controller.

The standard RST controller sensitivity function is

$$
S_{y r}=\frac{T\left(z^{-1}\right) B\left(z^{-1}\right)}{A\left(z^{-1}\right) S\left(z^{-1}\right)+B\left(z^{-1}\right) R\left(z^{-1}\right)}
$$

Sensitivity function from input $p(t)$ to output $y(t)$ is 


$$
S_{y p}=\frac{A\left(\mathrm{z}^{-1}\right) S\left(z^{-1}\right)}{A\left(z^{-1}\right) S\left(z^{-1}\right)+B\left(z^{-1}\right) R\left(z^{-1}\right)}
$$

Denote the polynomial $P\left(z^{-1}\right)=A\left(z^{-1}\right) S\left(z^{-1}\right)+B\left(z^{-1}\right) R\left(z^{-1}\right)$. The polynomial $S\left(z^{-1}\right)$ and $R\left(z^{-1}\right)$ can be determined by $P\left(z^{-1}\right)$. The desired leading poles is determined by the poles of controlled object.

From (7), polynomial $B\left(z^{-1}\right)$ has a zero at $z=1$. Considering eliminate ill effects of this zero, $B\left(z^{-1}\right)$ is supposed to be the divisor of $P^{\prime}\left(z^{-1}\right)$. Let $S\left(z^{-1}\right)=B\left(z^{-1}\right) S^{\prime}\left(z^{-1}\right)$ the equation can be more simplified to $P_{m}\left(z^{-1}\right)=S^{\prime}\left(z^{-1}\right)+R^{\prime}\left(z^{-1}\right)$. The locations of auxiliary poles are required to be close to the origin of $\mathrm{z}$-domain to reduce the effect of auxiliary poles at low frequency. Take a couple of auxiliary poles $P m=\left(1-0.5 z^{-1}\right)^{2}$ The polynomial $S^{\prime}\left(z^{-1}\right)$ and $R^{\prime}\left(z^{-1}\right)$ is obtained by solving Bézout's identity [16]. The corresponding sensitivity function is shown in Fig. 5.

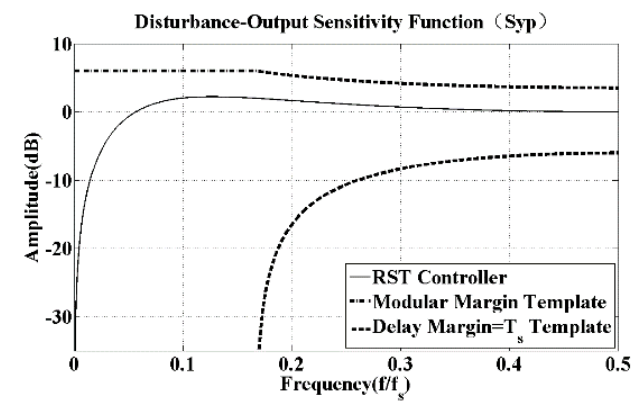

(a) High frequency

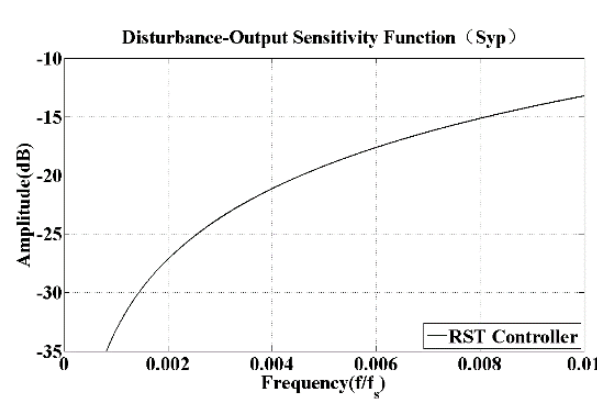

(b) Low frequency

Fig. 5. Sensitivity function curve from disturbance to output.

As shown in Fig. 5, the robustness requirement has been met, whereas the performance of platform cannot meet the demands. The amplitude of sensitivity function is more than $-30 \mathrm{~dB}$ at the frequency lower than $5 \mathrm{~Hz}$.

To satisfy all the requirements, the location of the auxiliary poles $\mathrm{z}=0.1$. A zero-poles resonator filter is introduced to the system, the filter is

$$
F_{s}=\frac{S_{i}}{P_{i}}=\frac{s^{2}+2 \zeta_{1} \omega_{0} s+\omega_{0}^{2}}{s^{2}+2 \zeta_{2} \omega_{0} s+\omega_{0}^{2}}
$$

where $\zeta_{1}=0.25, \zeta_{2}=0.5, \omega_{0}=10 \pi$.

After discretization, $S_{i}$ and $P_{i}$ are introduced as a divisor to $S^{\prime}\left(z^{-1}\right)$ and $P\left(z^{-1}\right)$. Re-calculate the Bézout's identity and update the $S^{\prime}\left(z^{-1}\right)$ and $R^{\prime}\left(z^{-1}\right)$. The sensitivity function curve after amendment is shown in Fig. 6.

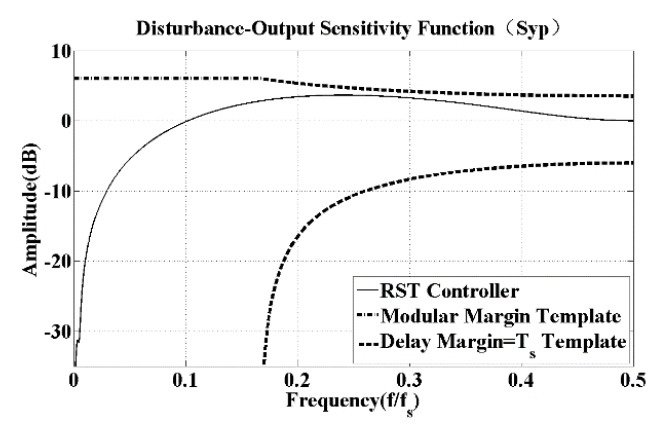

(a) High frequency

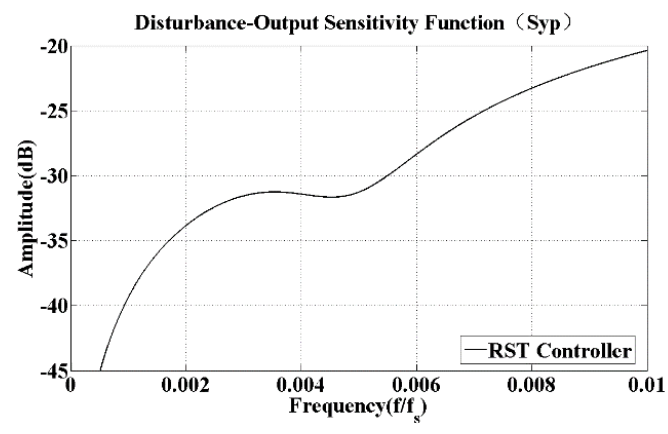

(b) Low frequency

Fig. 6. Sensitivity function curve from disturbance to output after correction. 
As shown in Fig. 6, through the adjustment of auxiliary poles and the introduction of zero-pole resonant filter, the robustness is weakened. The performance of the platform at low frequency meet the requirement, and the amplitude of isolation is no more than $-30 \mathrm{db}$. The requirement of robustness is also met.

\section{Validation and Comparison}

To validate the controller presented in this paper, simulations are taken in Matlab/Simulink. The comparison with classic PID controller is also carried out.

When the input signal is unit step signal, comparison of response under RST controller and PID controller is presented in Fig. 7. As shown in Fig. 7, the response time of inner modular RST controller is reduced to about $0.02 \mathrm{~s}$, while the response time of PID controller is up to $0.05 \mathrm{~s}$.

When the input signal is sine signal with amplitude of $1 \%$ s and frequency of $1 \mathrm{~Hz}$, comparison of response under RST controller and PID controller is presented in Fig. 8.

As shown in Fig. 8, tracking error of PID controller is $0.019 \%$, however the tracking error of inner modular RST controller is stabilized at about $0.006 \%$.

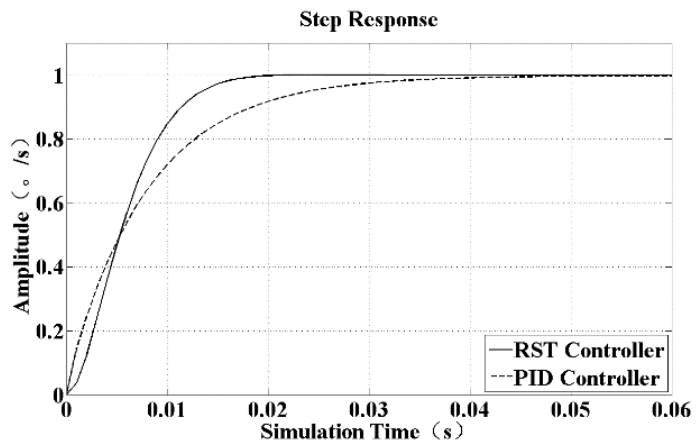

Fig. 7. Step response.

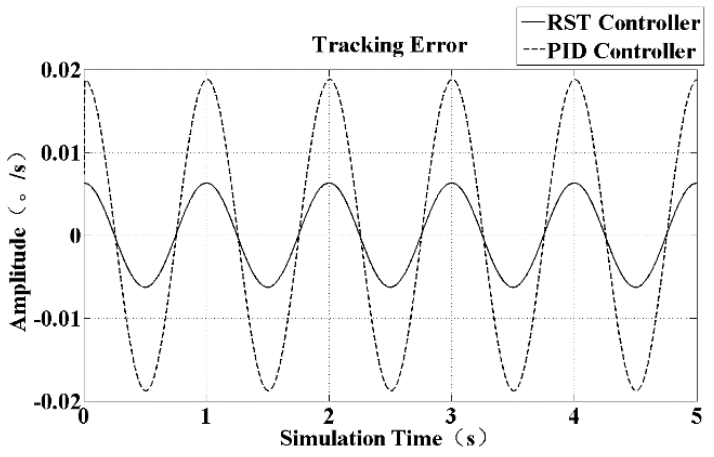

Fig. 8. Tracking error.

The sensitive function curve of PID controller and inner modular RST controller are shown in Fig. 9.

As is shown in Fig. 9, the ability of RST controller presented in this paper for suppressing disturbance signal has been much improved in low frequency. In main working frequency band, inhibitory ability improved by about $10 \mathrm{~dB}$. The rejection band for $-30 \mathrm{~dB}$ has improved from $1.8 \mathrm{~Hz}$ to $5.8 \mathrm{~Hz}$. At the high frequency section, PID controller and inner modular RST controller are both meet the robustness requirement.

The sinusoidal disturbance signal with frequency of $5 \mathrm{~Hz}$ and amplitude of $1 \% \mathrm{~s}$ is taken as an example, the output under influence of disturbance signal is shown in Fig. 10. The isolation of platform using PID controller is about $-20.91 \mathrm{~dB}$, whereas the isolation using inner module RST controller is about $-31 \mathrm{~dB}$. 


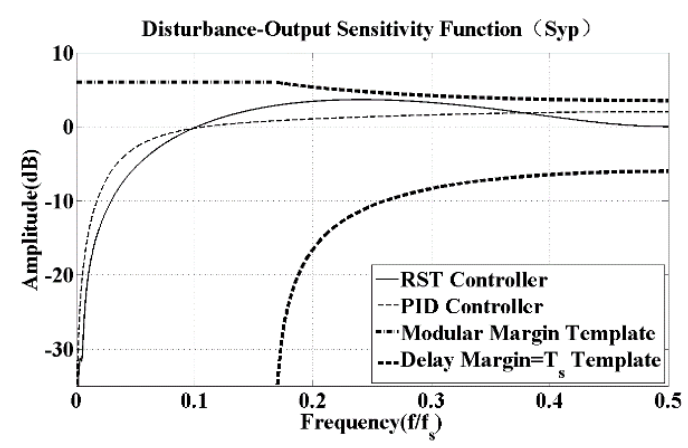

(a) High frequency

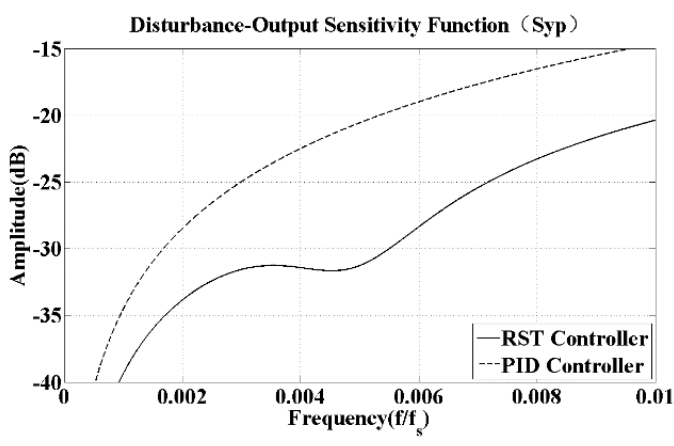

(b) Low frequency

Fig. 9. Sensitivity function comparison.

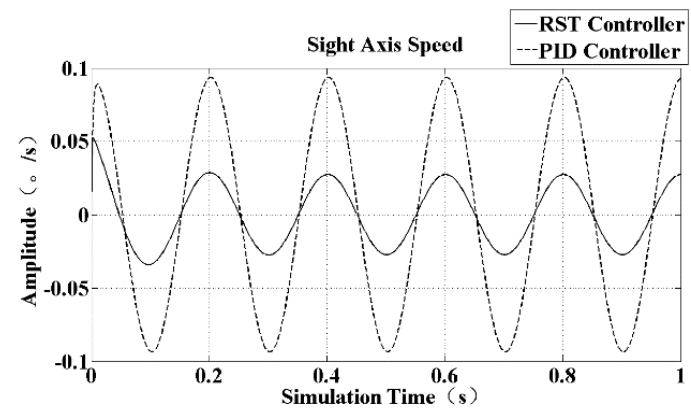

Fig. 10. Response of $5 \mathrm{~Hz}$ disturbance signal.

From the simulation results, the presented inner modular RST controller meet all the requirement of the platform with the presented sensitivity function template. With the presented inner modular RST controller the response time of the close loop system reduces from $0.05 \mathrm{~s}$ to $0.02 \mathrm{~s}$, and the tracking error of the sinusoidal signal is reduced to $0.6 \%$.

\section{Conclusion}

In this paper, a stable platform driven by USMs is taken into consideration. The inner gimbal is taken as an example to design the control system to reject the disturbance. A high performance controller is introduced, which can control the speed of the USM and suppress the high frequency disturbance. The simulation results validate the effectiveness of the presented controller. And the control performance is improved compared with the PID controller.

\section{Acknowledgements}

This work has been supported by the National Natural Science Foundation of China (No.51575260) and the Fundamental Research Funds for the Central Universities (NS20160001).

\section{Reference}

[1] White, F. (1974). Stabilized platform.

[2] Yuan, X. (2012). Foreign Electronic Measurement Technology, 9.

[3] Zhao, C. S. (2011). Ultrasonic Motors. Springer Berlin Heidelberg.

[4] Zeng, J. S., Yao, Z. Y., \& Zhao, C. S. (2005). China Small Motor Technology Conference.

[5] Pang, X. L., et al. (2008). Journal of Dynamics and Control, 6(1), 88-91.

[6] Hasturk, O., Erkmen, A. M., \& Erkmen, I. (2011). Lecture Notes in Engineering \& Computer Science. Springer Berlin Heidelberg. 
[7] Abdo, M. M., et al. (2014). ISA T., 53(2), 591-602.

[8] Xia, Y. X., et al. (2013). Control and Decision, 7, 1117-1120.

[9] Izuno, Y., et al. (1992). IEEE T. IND. APPL., 28(3), 613-618.

[10] Yildirim, S. (2005). J. INTELL. ROBOT SYST., 44(3), 247-261.

[11] Chen, T. C., et al. (2008). ISA T., 47(3), 325-38.

[12] Zhang, P., et al. (2011). Modeling and Control of Airborne/Missile-Borne Vision-Guidance Stabilized Platform

[13] Giraud, F., et al. (2003). EUR. PHYS. J-APPL. PHYS., 21,151-159.

[14] Shi, J., \& You, D. (2013). Ultrasonics, 54(2), 725-30.

[15] Landau, I. D., \& Zito, G. (2006). Digital Control Systems. Springer-Verlag New York, Inc.

[16] Shub, M., \& Smale, S. (2014). THEOR. COMPUT. SCI., 133(1), 141-164.

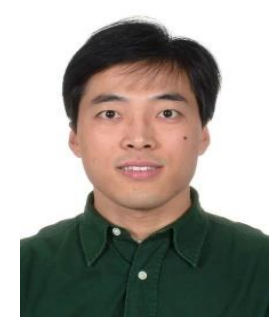

and control.

Pan Song was born in Zouping, China, in 1978. He received the B.S. degree in mechanical engineering from Shandong University of Technology, Zibo, China, in 2003, and the Ph.D. degree in mechanical engineering from Nanjing University of Aeronautics and Astronautics, Nanjing, China, in 2009. He is currently a lecturer in the College of Aerospace Engineering, Nanjing University of Aeronautics and Astronautics, Nanjing, China. His main research interests include piezo motor design, ultrasonic motor driving

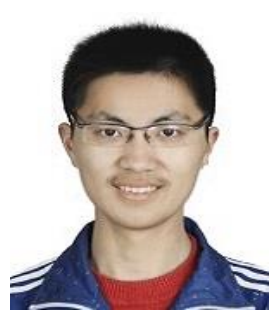

Xu Zhangfan was born in Rugao, China, in 1995. He received the B.S. degree from Nanjing University of Aeronautics and Astronautics Nanjing (NUAA), China, in 2016. He is currently a Ph.D. student in the College of Aerospace Engineering of Nanjing University of Aeronautics and Astronautics, Nanjing, China. His research interests include the structure and mechanics of piezoelectric motor and its applications.

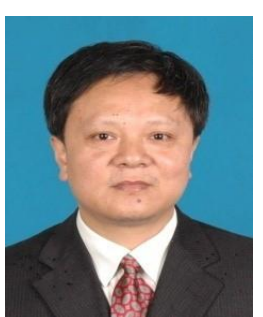

Huang Weiqing was born in Nantong, China, in 1963. He received the B.S. and M.S. degrees in mechanical engineering from Nanjing University of Aeronautics and Astronautics, Nanjing, China, in 1978, and 1990, respectively, and the Ph.D. degree in mechanical engineering from Hong Kong University of Science and Technology, Hong Kong, China, in 1992. He is currently a professor in Guangzhou University, Guangzhou, China. His current research interests include precision driving, ultrasonic machining and piezoelectric actuators. 